\title{
EDITORIAL
}

\section{MMPs are regulatory enzymes in pathways of inflammatory disorders, tissue injury, malignancies and remodelling of the lung}

\section{J. Müller-Quernheim}

$\mathbf{M}$ embers of the matrix metalloproteinase (MMP) family of zinc-dependent proteolytic enzymes regulate physiological and pathophysiological events in development, injury and repair, such as morphogenesis, vasculogenesis, remodelling of the extracellular matrix, cell migration, cleavage of cytokines, and activation of mediators and defensins. They are synthesised as secreted or transmembrane proenzymes and processed to their active forms by the removal of amino-terminal propeptides. Their activation by the cleavage of the prodomain from the latent proenzyme and the presence of natural inhibitors, such as tissue inhibitors of metalloproteinases (TIMPs), are important mechanisms that restrict the action of secreted MMPs to the micromilieu of their origin. This tight control of activation and the redundant design of the MMP system, points to their importance in the named processes. In most tissues, their constitutive expression is low and their overexpression at sites of inflammation or other pathogenic events was thought to be due the classical role of extracellular matrix degradation.

However, MMPs are no longer thought of as just matrixdegrading proteins. Rather, proteolysis should be viewed as an important post-translational principle of regulating a fast spectrum of biological processes, and the functional redundancy in combination with a wide substrate overlap underscores the pivotal roles of MMPs not only as effectors but also in regulatory mechanisms. Thus, MMPs are essential in many physiological events and the increasing interest in their function originates from their contribution to inflammatory and remodelling processes, in which they regulate physical barriers, release bioactive factors, participate in membrane shedding, modulate chemokine gradients that regulate leukocyte motility, activate cells via triggering of proteinaseactivated receptors (PAR), and alter the activity status of other proteases, including other MMPs. Consequently, the majority of MMP substrates are non-matrix proteins, which underscores their critical roles in regulatory processes $[1,2]$. Reports implicating MMPs in destructive pathogenic processes of pulmonary disorders are found in the literature from the early 1990s; more recently it has been recognised that the clinical

CORRESPONDENCE: J. Müller-Quernheim, Dept of Pneumology, University Medical Center Freiburg, Killianstraße 5, 79106 Freiburg, Germany. E-mail: joachim.mueller-quernheim@uniklinikfreiburg.de outcome of these processes is the result of an imbalance between MMP action and its inhibitors.

In humans, 24 MMPs have been identified and a cluster of nine MMP genes is located on chromosome 11q22, which are likely to be derivative of a single ancestor gene. The $24 \mathrm{MMP}$ genes can be found on 10 different human chromosomes and their gene products are integrated in a redundant network, which can be seen by analysis of murine MMP knockout mutants. The phenotypes of these mutants are surprisingly benign, with pathologies in growth and ossification indicating functional redundancy and a rather limited activity in health [3]. Proteolysis, such as cleavage by MMPs, is a very effective mechanism which enables an organism to react very rapidly to pathologic events. Several types of lung cells, such as macrophages, neutrophils, epithelial cells and stroma cells, are capable of releasing MMPs in response to endogenous or exogenous stimuli, leading to complex roles of MMPs in health and disease; this is the topic of a new series that commences in the current issue of the European Respiratory Journal (ERJ) with a review by LÖFFEK et al. [3], which provides an overview of the physiological roles of MMPs. The ERJ has always disseminated advances in knowledge about MMPs and commits to doing so consistently. To this end, in the forthcoming months, the ERJ's new series on MMPs will provide in-depth coverage of the biological role of MMPs in pulmonary health and distinct disorders. Tuberculosis, cystic fibrosis (CF), acute lung injury (ALI), adult respiratory distress syndrome (ARDS), asthma, chronic obstructive pulmonary disease (COPD), idiopathic pulmonary fibrosis (IPF), lung cancer, pulmonary hypertension, and MMPs as drug targets will be addressed.

Fibrillar collagens, which are highly resistant to enzymatic cleavage, are important constituents of the pulmonary extracellular matrix. However, collectively, MMPs are able to degrade all components of the extracellular matrix, resulting in cavities [4], and about a decade ago it was recognised that lung extracellular matrix destruction by MMPs was essential to tuberculosis [5]. The destruction of extracellular matrix by MMPs allows mycobacteria to spread from the interstitium to the airways, and results in cavities with an immunodeficient milieu in which mycobacteria can proliferate as a consequence of a missing protective immune response. These events are initiated by mycobacterial MMP activation within the monocytes/macrophages hosting the mycobacteria in the pulmonary 
interstitium, as discussed by ELKINGTON et al. [6] in a forthcoming article in this series, and elsewhere [7].

Animal and clinical studies demonstrate an increased expression of MMPs in ALI and ARDS. However, different MMP and TIMP levels are found over time. Their role in destructive pathologies and tissue repair needs to be balanced in order to identify new therapeutic intervention points for these devastating disorders, as the role they play as pathogenic factors or compensating mechanisms remains to be established. This is considered in a later article in the series by DAVEY et al. [8].

Increased expression of MMPs has been demonstrated in almost all inflammatory diseases, including IPF [9]. A microarray study previously demonstrated that MMP-7 was the most significantly overexpressed gene in IPF and that MMP-7deficient mice were protected from bleomycin-induced fibrosis [10]. However, MMP-7 and its inhibitor are not only found in IPF but also in cryptogenic organising pneumonia, a disease which generally responds well to anti-inflammatory therapy. These observations lead to the hypothesis that MMP-7 overexpression represents an attempt of the body to control the fibrogenic processes, rather than being a pathogenic factor [11]. A better understanding of these processes is necessary for the development of new treatment strategies manipulating the biology of MMPs, as discussed by DANCER et al. [12] later in the series, and elsewhere [13].

Similar considerations, whether MMP overexpression is a pathogenic mechanism or a compensating response of the body, apply to CF. For pulmonary CF manifestations, however, abundant evidence points to a pathogenic role [14] and clinical studies targeting the protease/anti-protease imbalance can now be designed along the lines of pharmacological antielastase studies performed in CF [15].

Around 2,000 years ago, Aulus Cornelius Celsus described the classical symptoms of inflammatory tissue responses to harmful stimuli as rubor (redness), tumor (swelling), dolor (pain), and calor (heat), which are the result of the exudation of plasma proteins, degradation of extracellular matrix, activation of mediators, and recruitment of leukocytes, as observed in the airways of patients with asthma and COPD. MMPs have shown to be critically involved in all these processes [16, 17], and in particular phenotypes presenting with the overlap of asthma and COPD are associated with risk alleles of variant MMP genes with differences in the expression of their gene products $[18,19]$.

In lung cancer, the expression of a number of MMPs and their inhibitors is exaggerated and may be causally linked to enhanced tumour progression and metastasis [20-23]. This will be the focus of a later instalment in the series by H. MCGARRY and N. KAMINSKI.

Unsurprisingly, this broad involvement of MMPs in the pathologies of pulmonary disorders has developed interest in pharmacological manipulation of the balance between MMPs and their inhibitors, including TIMPs, and several established drugs have been shown to influence MMP activity. In this context, it is interesting to note that p-amino salicylic acid, a drug used to treat tuberculosis, is derived from the original cyclooxygenase inhibitor salicylic acid and inhibits mycobacteriadriven prostaglandin $\mathrm{E}_{2}$ accumulation, thereby suppressing
MMP-1 secretion. This mode of drug behaviour blocks the mycobacterial exploitation of MMP-1 for tissue destruction and cavity formation [24]. Furthermore, doxycycline, an antimicrobial drug with some immunomodulatory behaviour, has been shown to inhibit MMP-9, in part explaining its non-antimicrobial actions [25]. MMP-2 and MMP-9 expression can be reduced by pentoxifyline to 30 and $56 \%$, respectively [26]; this to some extent explains the immunomodulatory action of this drug. Other inhibitors of MMP-9 preventing adverse remodelling are the angiotensin converting enzyme (ACE) inhibitors, which act on zinc-dependent endopeptidases such as ACE and MMP-9 $[27,28]$. These molecules may be the basis for more specific MMP inhibitors to come. Unfortunately, studies using currently available MMP inhibitors have not reached their therapeutic endpoints, but third-generation MMP inhibitors are in development that will specifically inhibit individual MMPs to manipulate the MMP/MMP inhibitor imbalance. This is further discussed in the series article by VANDENBROUCKE et al. [29].

The series on MMPs in health and disease that begins in this issue of the ERJ aims to summarise current knowledge on the importance of these molecules for lung health. A more detailed understanding of physiologic and pathophysiologic roles of MMPs will have a substantial impact on the pathogenic concepts of most pulmonary disorders, which will influence our therapeutic approach. MMP research will gain momentum in the near future and this series offers a basis for a thorough discussion of future advances.

\section{STATEMENT OF INTEREST}

None declared.

\section{REFERENCES}

1 Parks WC, Wilson CL, Lopez-Boado YS. Matrix metalloproteinases as modulators of inflammation and innate immunity. Nat Rev Immunol 2004; 4: 617-629.

2 Li Q, Park PW, Wilson CL, et al. Matrilysin shedding of syndecan-1 regulates chemokine mobilization and transepithelial efflux of neutrophils in acute lung injury. Cell 2002; 111: 635-646.

3 Löffek S, Schilling O, Franzke C-W. Biological role of matrix metalloproteinases: a critical balance. Eur Respir J 2011; 38: 191-208.

4 Brinckerhoff CE, Matrisian LM. Matrix metalloproteinases: a tail of a frog that became a prince. Nat Rev Mol Cell Biol 2002; 3: 207-214.

5 Price NM, Farrar J, Tran TT, et al. Identification of a matrixdegrading phenotype in human tuberculosis in vitro and in vivo. J Immunol 2001; 166: 4223-4230.

6 Elkington PT, Ugarte-Gil CA, Friedland JJ. Matrix metalloproteinases in tuberculosis. Eur Respir J 2011; (in press).

7 Volkman HE, Pozos TC, Zheng J, et al. Tuberculous granuloma induction via interaction of a bacterial secreted protein with host epithelium. Science 2010; 327: 466-469.

8 Davey A, McAuley DF, O'Kane CM. Matrix metalloproteinases in acute lung injury: mediators of lung injury and drivers of repair. Eur Respir J 2011; (in press).

9 McKeown S, Richter AG, O'Kane C, et al. MMP expression and abnormal lung permeability are important determinants of outcome in IPF. Eur Respir J 2009; 33: 77-84.

10 Zuo F, Kaminski N, Eugui E, et al. Gene expression analysis reveals matrilysin as a key regulator of pulmonary fibrosis in mice and humans. Proc Natl Acad Sci USA 2002; 99: 6292-6297.

11 Huh JW, Kim DS, Oh YM, et al. Is metalloproteinase-7 specific for idiopathic pulmonary fibrosis? Chest 2008; 133: 1101-1106. 
12 Dancer RCA, Wood AM, Thickett DR. Metalloproteinases in idiopathic pulmonary fibrosis. Eur Respir J 2011; (In press).

13 Kamio K, Liu XD, Sugiura $H$, et al. Statins inhibit matrix metalloproteinase release from human lung fibroblasts. Eur Respir J 2010; 35: 637-646.

14 Gaggar A, Hector A, Bratcher PE, et al. The role of matrix metalloproteases in cystic fibrosis lung disease. Eur Respir J 2011; [Epub ahead of print DOI: 10.1183/09031936.00173210].

15 Griese M, Kappler M, Gaggar A, et al. Inhibition of airway proteases in cystic fibrosis lung disease. Eur Respir J 2008; 32: 783-795.

16 Botelho FM, Nikota J, Bauer C, et al. A mouse GM-CSFR antibody attenuates neutrophilia in mice exposed to cigarette smoke. Eur Respir J 2011; [Epub ahead of print DOI: 10.1183/09031936.00076210].

17 Roberts ME, Magowan L, Hall IP, et al. Discoidin domain receptor 1 regulates bronchial epithelial repair and matrix metalloproteinase production. Eur Respir J 2011; 37: 1482-1493.

18 Hunninghake GM, Cho MH, Tesfaigzi Y, et al. MMP12, lung function, and COPD in high-risk populations. N Engl J Med 2009; 361: 2599-2608.

19 Lee SY, Kim MJ, Kang HG, et al. Polymorphisms in matrix metalloproteinase- $-1,-9$ and -12 genes and the risk of chronic obstructive pulmonary disease in a Korean population. Respiration 2010; 80: 133-138.

20 Liu D, Nakano J, Ishikawa S, et al. Overexpression of matrix metalloproteinase-7 (MMP-7) correlates with tumor proliferation, and a poor prognosis in non-small cell lung cancer. Lung Cancer 2007; 58: 384-391.
21 Ishikawa S, Takenaka K, Yanagihara K, et al. Matrix metalloproteinase-2 status in stromal fibroblasts, not in tumor cells, is a significant prognostic factor in non-small-cell lung cancer. Clin Cancer Res 2004; 10: 6579-6585.

22 Qian $\mathrm{Q}$, Wang $\mathrm{Q}$, Zhan $\mathrm{P}$, et al. The role of matrix metalloproteinase 2 on the survival of patients with non-small cell lung cancer: a systematic review with meta-analysis. Cancer Invest 2010; 28: 661-669.

23 Scherf DB, Dally H, Muller P, et al. Single nucleotide polymorphisms in matrix metalloproteinase genes and lung cancer chemotherapy response and prognosis. Eur Respir J 2010; 35: 381-390.

24 Rand L, Green JA, Saraiva L, et al. Matrix metalloproteinase- 1 is regulated in tuberculosis by a p38 MAPK-dependent, p-aminosalicylic acid-sensitive signaling cascade. J Immunol 2009; 182: 5865-5872.

25 Ren S, Guo LL, Yang J, et al. Doxycycline attenuates acroleininduced mucin production, in part by inhibiting MMP-9. Eur J Pharmacol 2011; 650: 418-423.

26 Deree J, Martins J, de Campos T, et al. Pentoxifylline attenuates lung injury and modulates transcription factor activity in hemorrhagic shock. J Surg Res 2007; 143: 99-108.

27 Yamamoto D, Takai S. Pharmacological implications of MMP-9 inhibition by ACE inhibitors. Curr Med Chem 2009; 16: 1349-1354.

28 Yamamoto D, Takai S, Jin D, et al. Molecular mechanism of imidapril for cardiovascular protection via inhibition of MMP-9. J Mol Cell Cardiol 2007; 43: 670-676.

29 Vandenbroucke RE, Dejonckheere E, Libert C. A therapeutic role for MMP inhibitors in lung diseases? Eur Respir J 2011; (in press). 